\title{
Trade-offs em Serviços Customizados e o Ponto de Vista do Cliente
}

\section{Trade-offs in Customized Services and the Customer Point of View}

\begin{abstract}
Rafael Teixeira*
Doutorando em Administração pela Texas A\&M University, Estados Unidos.
\end{abstract}

Ely Laureano Paiva

Doutor em Administração pela UFRGS.

Professor do PPGA/UNISINOS, São Leopoldo/RS, Brasil.

*Endereço: Rua São Manoel, 1300 / 202, Porto Alegre/RS, 90620-110. E-mail: teixeira.rafa@gmail.com 


\title{
ResUMo
}

Os trade-offs em estratégia de operações podem ser descritos como as incompatibilidades operacionais existentes entre diferentes critérios competitivos. Serviços customizados implicam entregas mais demoradas e custos de produção mais elevados do que serviços padronizados. Considerando que as preferências dos clientes terão repercussões para as escolhas estratégicas dos critérios competitivos e para os trade-offs da área de operações, esta pesquisa buscou avaliar as relações entre os atributos customização, tempo de entrega e preço baixo, sob o ponto de vista do cliente, verificando a ocorrência dos tradeoffs. Estudos que envolvem a existência e razões desses possíveis trade-offs têm sido recorrentes na literatura; porém pouca atenção tem sido colocada na influência dos clientes para essa questão. Para isso foi realizada um levantamento survey com 243 clientes de serviços customizados de comunicação de dados de uma operadora de telecomunicações para avaliação da importância de cada um destes atributos. Os resultados mostraram que o trade-off entre customização e tempo de entrega foi pouco relevante no caso estudado. Por outro lado, os resultados sugerem que o trade-off entre customização e custos é relevante para o serviço analisado.

Palavras-chave: critérios competitivos; trade-offs; customização.

\begin{abstract}
Trade-offs in operations strategy can be defined as the performance incompatibility between two or more different competitive criteria. Customized services imply slower delivery time and high production costs than in standardized services. Considering that customer's preferences will affect the strategic decisions about competitive criteria and trade-offs, this research aimed to evaluate customization, delivery speed and low price attributes from the customer's perspective, analyzing the trade-offs among them. The literature has put little emphasis on the influence of the customers over trade-offs. In order to assess the importance of each service's attribute, a survey was applied to 243 customers a data communication customized service provided by a tellecommunication company. The results indicated that trade-offs analyzed do not present the relations expected, especially between customization and delivery speed. On the other hand, the results suggest that the tradeoff between customization and costs is relevant for the service analyzed.
\end{abstract}

Key words: competitive criteria; trade-offs; customization. 


\section{INTRODUÇÃO}

Desde Skinner (1969) que, no final da década de 60, chamou a atenção para a contribuição estratégica da área de produção, a área de operações tem discutido significativamente seu papel na estratégia das empresas. Complementarmente ao trabalho seminal de Skinner (1969), Wheelwright (1984) propôs os critérios competitivos que orientam a estratégia a ser seguida pela área de operações. Desde então, a discussão sobre os critérios competitivos tem sido recorrente na área (Corbett \& Wasenhove, 1993; Ferdows \& De Meyer, 1990; New, 1992; Noble, 1995).

Tanto Skinner (1974) como Wheelwright (1984) destacaram que seria difícil uma organização obter elevado desempenho em cada um dos critérios ao mesmo tempo. Para eles, a organização teria de optar por competir em certos critérios competitivos, fazendo, assim, escolhas estratégicas. Essa necessidade de escolher em quais critérios competir estaria relacionada aos trade-offs da área de operações.

Os trade-offs da área podem ser caracterizados como "incompatibilidade entre dois ou mais critérios, ou seja, as situações em que a melhoria de um critério poderá implicar impacto negativo em outro" (Paiva, Carvalho, \& Fensterseifer, 2004, p. 55). Assim, por exemplo, New (1992) defende a existência do trade-off entre customização e tempo de entrega.

Outra corrente de pesquisadores, contudo, tem sugerido a inexistência dos tradeoffs. Baseados em modelos acumulativos de competências, nos quais os critérios competitivos estariam relacionados, eles argumentam que muitos trade-offs não existiriam (Corbett \& Wasenhove, 1993; Ferdows \& De Meyer, 1990; Silveira \& Fogliatto, 2002). Porém, mesmo com esses estudos, trabalhos complementares têm buscado evidências empíricas da existência dos trade-offs na área de operações (Boyer \& Lewis, 2002; New, 1992), particularmente os que envolvem customização, tempo de entrega e preço (Ahlström \& Westbrook, 1999; Mapes, New, \& Szwejczewski, 1997; New, 1992; Paiva et al., 2004; Schmenner \& Swink, 1998).

Por outro lado, pouca atenção tem sido dada à participação do cliente diante dos dilemas que envolvem os critérios competitivos, pois poucos trabalhos foram desenvolvidos com essa orientação. Ao mesmo tempo, a inclusão do cliente é fundamental porque, dependendo das suas preferências sobre determinados atributos de um produto, haverá reflexos no desempenho dos critérios 
competitivos que a área de operações deverá ter como meta (Bardacki \& Whitelock, 2003).

Considerando-se tais proposições, o problema de pesquisa que emerge é este: Quais as relações entre os atributos customização, tempo de entrega e preço, sob a ótica dos clientes? Dependendo das preferências dos clientes em relação a estes atributos, a área de operações teria de incorrer nestes trade-offs? Para responder a essas questões, esta pesquisa tem como objetivos identificar a importância da customização, do tempo de entrega e do preço e verificar se as relações entre estes atributos poderiam influenciar os trade-offs na área de operações.

\section{REVISÃo dA LiterATURA}

\section{Os Critérios Competitivos}

O conceito de estratégia em operações está relacionado com o papel da área de operações na definição da estratégia das organizações e, como consequiência, nas definições dos critérios competitivos em que a empresa pretende competir (Skinner, 1969, 1974). Uma das vantagens na definição dos critérios competitivos é compreender seus reflexos nas decisões estruturais e táticas da organização (Wheelwright, 1984). Por exemplo, priorizar a competição em custo poderá implicar arranjos de capacidade, tecnologia e força de trabalho, que seriam diferentes, se a prioridade competitiva fosse outra, como variedade de produto ou customização.

Os critérios competitivos mais usuais na literatura são: custo, qualidade, confiabilidade e flexibilidade. Inicialmente propostos por Wheelwright (1984), eles tornaram-se referência na literatura de operações e atualmente são citados por muitos outros autores (New, 1992; Noble, 1995; Slack, Chambers, Harland, Harrison, \& Johnston, 1999; Verman \& Thompson, 1999). Alguns autores utilizam variações destes critérios (Hayes \& Pisano, 1996; Mapes et al., 1997), acrescentando, por exemplo, tempo de entrega, embora muitos destes critérios possam ser classificados dentro daqueles propostos por Wheelwright (1984).

De forma sucinta, o critério competitivo qualidade significa prover um produto de alta qualidade ou que tenha características ou desempenho que não estão disponíveis nos outros produtos do mercado (Wheelwright, 1984). Para Slack et al. (1999, p. 59), qualidade significa "fazer as coisas certas". 
O critério flexibilidade pode considerar dois aspectos: flexibilidade de produto e de volume (Wheelwright, 1984). A flexibilidade de produto enfatiza a produção de produtos não padronizados e a liderança na introdução de novos produtos. A flexibilidade de volume enfatiza a habilidade em acelerar ou desacelerar a produção rapidamente. Slack et al. (1999) propõem ainda a flexibilidade de entrega, onde se buscam diferentes tempos de entrega, e de mix de produtos, com ampla variedade de composto de produtos.

A customização pode ser entendida como a flexibilidade levada ao seu extremo, na qual se busca a capacidade de produzir produtos customizados com os benefícios próximos aos da produção em massa (Pine, 1994). Os clientes têm papel fundamental para o sucesso da customização e são colocados em papel de destaque nesse processo (Broekhuizen \& Alsem, 2002; McCutcheon, Raturi, \& Meredith, 1994; Pine, 1994; ). Além disso, importa destacar que a modularização de peças e componentes viabiliza economicamente a customização (Pine, 1994; Swaminathan, 2001).

O critério competitivo confiabilidade se relaciona com a confiança de que os produtos funcionarão conforme as especificações, com as entregas no tempo prometido e com o reparo rápido de um problema pelo fornecedor (Wheelwright, 1984). Alguns autores consideram a entrega um critério competitivo separado da confiabilidade (New, 1992). Desse modo, existiria importante distinção entre confiabilidade na entrega e tempo de entrega. Confiabilidade na entrega é entregar os produtos dentro do prazo prometido, enquanto a velocidade no tempo de entrega é entregar os produtos mais rapidamente (Paiva et al., 2004). Para Slack et al. (1999, p. 62), "rapidez significa quanto tempo os consumidores precisam esperar para receber seus produtos ou serviços". A rapidez no tempo de entrega é vista por diversos autores como fonte de vantagem competitiva (Stalk, 1998), pois pode proporcionar reduções de custos, entregas confiáveis, flexibilidade, agilidade, redução de estoques, qualidade e racionalização de processos (Rohr \& Correa, 1998; Wheelwright \& Clark, 1992).

O último critério competitivo visa à redução dos custos de produção, que envolvem, por exemplo, mão-de-obra, instalações e matéria-prima, para ofertar produtos a preços menores. Este critério também é tido como fonte de vantagem competitiva (New, 1992; Wheelwright, 1984 ) e está ligado aos preços dos produtos e serviços (Slack et al., 1999).

É válido destacar que os critérios competitivos estão relacionados com atributos de produtos e serviços, tais como preço e qualidade, podendo ser avaliados pelos clientes (Slack et al., 1999; Verma \& Thompson, 1999). 


\section{Os Trade-offs}

O conceito de trade-off tem sido muito abordado na literatura nas últimas décadas (Corbett \& Wasenhove, 1993; Hayes \& Pisano, 1996; Skinner, 1969, 1974). Usualmente, refere-se à necessidade da organização de escolher critérios competitivos para sustentar sua estratégia de negócios, embora alguns destes critérios possam ser "incompatíveis" operacionalmente (Wheelwright, 1984). Para Skinner (1974, p. 115), "uma fábrica não pode ter um bom desempenho em cada critério", devido a limitações de recursos. A escolha de um critério pode tornar inviável o elevado desempenho de outro critério. "É difícil (mas não impossível) e potencialmente perigoso para uma empresa tentar competir por um desempenho superior em todas estas dimensões simultaneamente" (Wheelwright, 1984, p. 81). Para uma melhor compreensão, a lógica dos trade-offs pode ser expressa por meio de uma função inversamente correlacionada entre duas variáveis (Hayes \& Pisano, 1996).

Isso indica que o elevado desempenho em uma variável implica, automaticamente, o desempenho mais baixo de outra. Essa relação traria implicações diretas para as decisões de estrutura e infra-estrutura da área de operações, conforme citado anteriormente.

É válido destacar que os trade-offs tornam-se importantes, devido às relações existentes entre os critérios competitivos e as decisões estruturais e táticas da área de operações (Mapes et al., 1997). Tão importante quanto escolher um critério competitivo é alinhar os recursos da organização para garantir que ele seja exeqüível (Wheelwright \& Hayes, 1985).

Contudo outra corrente de pesquisadores vem sugerindo um modelo alternativo para os trade-offs (Collins, Cordon, \& Julien, 1998; Corbett \& Wasenhove, 1993; Ferdows \& De Meyer, 1990). Eles se baseiam no modelo acumulativo de competências denominado de cone de areia (Ferdows \& De Meyer, 1990). Tal modelo pressupõe que os critérios competitivos estão relacionados entre si. Como conseqüência, o desempenho de um critério afetaria simultaneamente os demais, criando uma obtenção contínua de melhorias. Essas melhorias elevariam o desempenho de vários critérios e, conseqüentemente, implicariam a inexistência dos trade-offs.

Diversos estudos desenvolvidos questionaram a existência dos trade-offs na área de operações. Noble (1995) encontrou evidências que confirmam o modelo acumulativo de competências. Szwejczewski, Mapes e New (1997) testaram o trade-off entre tempo de produção e confiabilidade na entrega e concluíram sua inexistência. Silveira e Fogliatto (2002) analisaram grandes amostras que 
apóiam empiricamente o modelo acumulativo de competências. Entretanto, para acirrar a discussão, Mapes et al. (1997) verificaram que a variedade de produto apresenta uma relação negativa com outros critérios, enquanto que Boyer e Lewis (2002) encontraram trade-offs mesmo em modelos acumulativos de competências.

\section{Os Trade-offs em Customização}

Vários autores defendem a existência de alguns trade-offs, entre os quais aqueles que envolvem a customização de produtos e serviços. Para New (1992), muitos trade-offs deixaram de existir; porém o trade-off entre customização e tempo de entrega permanece. Nesse sentido, Mapes et al. (1997) analisaram o modelo acumulativo de competências e verificaram que a variedade do produto tem impacto negativo sobre o tempo de entrega. Schmenner e Swink (1998), ao proporem uma variação da matriz produto x processo, destacaram que a velocidade do fluxo de materiais aumenta, à medida que diminui a variabilidade inerente à customização. De forma complementar, McCutcheon et al. (1994) afirmaram que as empresas devem buscar elevado desempenho nos critérios customização e tempo de entrega, embora em algumas situações eles sejam incompatíveis. O trabalho desenvolvido por Salvador e Forza (2004) junto a empresas italianas mostrou que diversas empresas estão sendo pressionadas pelos clientes para entregar produtos customizados de forma cada vez mais rápida.

Além disso, o trade-off entre customização e tempo de entrega também é influenciado pelo tipo de produto a ser produzido. Produtos mais simples tenderão a ser customizados de forma mais rápida, enquanto produtos mais complexos tenderão a levar mais tempo para serem produzidos. Por exemplo, customizar um CD numa loja da cadeia Personic Music levará menos tempo do que customizar uma calça nas lojas Levi Strauss \& Co (Bardacki \& Whitelock, 2003). Outro fator que influencia o tempo de entrega é o nível de customização. Se o item a ser customizado estiver na etapa de montagem, a customização será mais rápida do que se ele estivesse no nível de design, no qual todo o projeto de um produto é customizado de acordo com as necessidades de um cliente (Paiva et al., 2004).

Baseada nessas características, a matriz de produto $\mathrm{x}$ processo pode ser adaptada para uma nova matriz, onde se destaca a relação entre a customização e o tempo de entrega (Figura 1). 


\section{Figura 1: Matriz Tempo de Resposta x Customização}

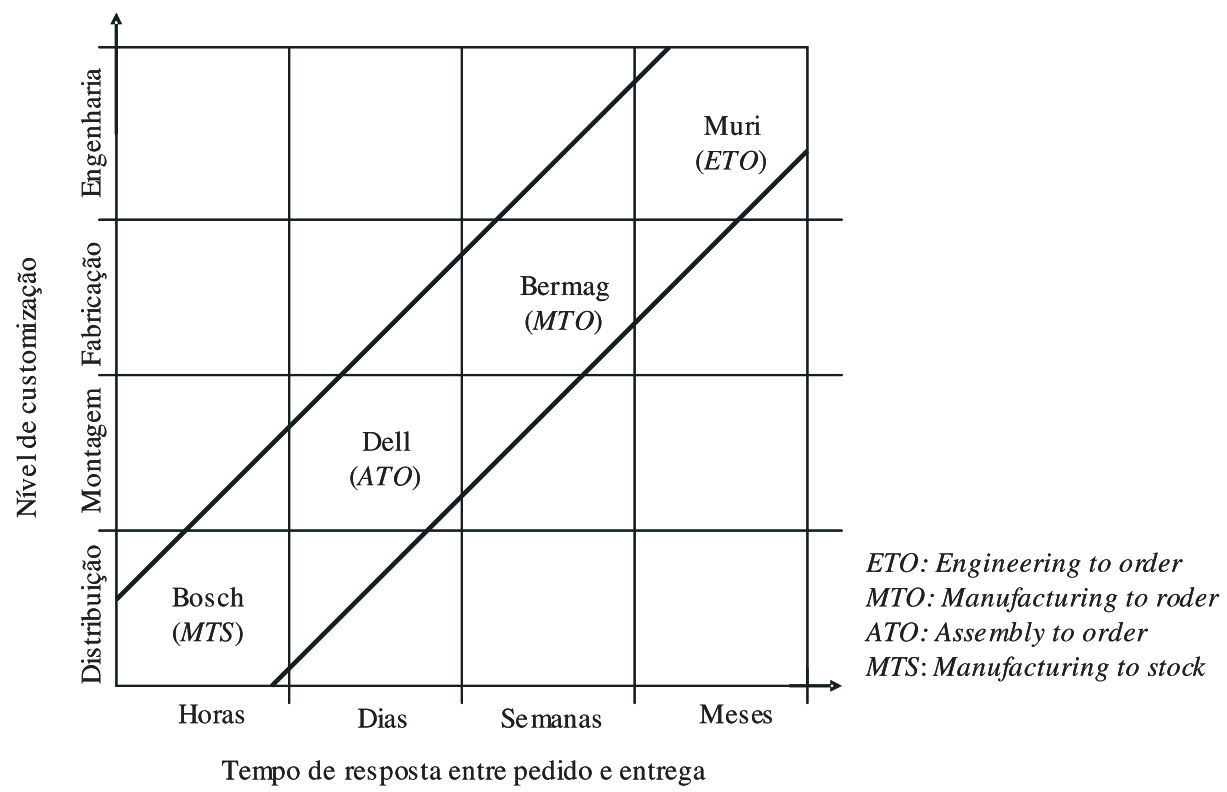

Fonte: Paiva et al. (2004).

Outros autores também mostram a existência do trade-off entre customização e custos, com reflexos para os preços de um produto. New (1992) defendeu a permanência deste trade-off, ao argumentar que a estratégia de customização eleva os custos de produção, devido à maior complexidade do sistema de produção. Efetivar a implantação da customização exige a inclusão de processos, máquinas, treinamento de mão-de-obra e estoques além daqueles requeridos pela padronização. Ahlström e Westbrook (1999) realizaram uma pesquisa com empresas britânicas cujos resultados indicaram que a pior consequiência da customização e, ao mesmo tempo, a maior barreira a ela era o aumento dos custos de produção. Levando em consideração que os custos têm relação direta com os preços (Slack et al., 1999), então a customização implicaria produtos e serviços com preços mais elevados.

Dentro da perspectiva do cliente, Bardacki e Whitelock (2003) crêem que os clientes estariam dispostos a pagar um preço mais elevado por produtos customizados, devido a um maior valor agregado, já que tais produtos se ajustam melhor às necessidades de cada cliente.

\section{A Importância dos Clientes para o Tema Relativo aos Trade-offs}

Uma das justificativas para o desenvolvimento deste trabalho encontra respaldo na própria característica dos trabalhos da área, ao abordar a questão dos trade- 
offs apenas sob a ótica da área de operações. Conforme destaca Heikkilä (2002), iniciar os esforços de melhoria na área de operações pelo envolvimento dos clientes não é prática usual dos gerentes de operações.

Todavia o cliente desempenha papel importante para a organização das operações. Para Bardacki e Whitelock (2003), as decisões dos clientes terão impacto na estratégia que será adotada pela área de operações.

A importância dos clientes para os trade-offs foi destacada por Silveira e Slack (2001). Segundo os autores, as expectativas/necessidades dos clientes podem ser consideradas como importante fator externo para a existência dos tradeoffs, dentro da área de operações. Os autores, ao conduzirem um estudo de caso múltiplo, constataram que o fator expectativas dos clientes, dentre diversos outros, era o único que aparecia em todas as empresas estudadas. Esse resultado pode ser considerado relevante, pois se deve ponderar que são os clientes que atribuem elevada ou reduzida importância a um par de critérios competitivos, o que os torna prioridade e gera, por consequiência, possíveis trade-offs operacionais.

Collins et al. (1998) também concordam que o desenvolvimento de critérios competitivos só tem sentido, quando está alinhado com as exigências e desejos dos clientes. Dentro dessa perspectiva, Verma e Thompson (1999) desenvolveram uma pesquisa para verificar a importância dada pelos clientes e gerentes aos critérios competitivos de uma rede de pizzarias. Os resultados mostraram diferenças entre o que os clientes e os gerentes valorizavam e evidenciaram uma falta de alinhamento entre o que os clientes efetivamente queriam e o que os gerentes pensavam que os clientes queriam.

\section{Metodologia}

Para operacionalização da pesquisa, deve-se destacar primeiramente que os critérios competitivos da área de operações têm relação direta com os atributos de um produto ou serviço (Corbett \& Wasenhove, 1993). Como exemplo, podese citar o trabalho de Verma e Thompson (1999), que utilizaram os atributos dos produtos e serviços de uma rede de pizzarias para avaliar seus critérios competitivos.

Assim, para avaliar a importância dos critérios competitivos customização, tempo de entrega e preço, sob o ponto de vista dos clientes foi realizada uma pesquisa de satisfação em relação ao desempenho de serviços customizados de comunicação de dados de uma operadora de telecomunicações. Os serviços de 
comunicação de dados são todos os serviços que têm a função de transmitir e receber dados de informações entre dois ou mais pontos distantes geograficamente. Eles são utilizados pelas empresas para conexão de informações dos bancos de dados de suas unidades que se encontram em diferentes localidades em um território. Dessa forma, os critérios competitivos da área de operações foram avaliados como atributos desses serviços prestados por uma operadora de telecomunicações.

O desenvolvimento da pesquisa de satisfação seguiu o modelo proposto Hayes (1997). A pesquisa foi dividida em duas etapas: uma exploratória e outra descritiva.

\section{Etapa Exploratória}

A etapa exploratória teve por objetivo gerar os indicadores (variáveis) para a construção do instrumento de coleta de dados da etapa descritiva. Este procedimento, além de estar de acordo com o proposto para uma pesquisa de satisfação, também segue o recomendado por Hinkin (1995), que defende uma abordagem qualitativa e indutiva para a geração dos itens de um instrumento de coleta de dados.

O grupo de entrevistados foi composto por dez clientes e dois executivos da empresa prestadora do serviço. Os entrevistados foram selecionados por julgamento, em que se procurou selecionar os clientes que já possuíssem serviços customizados de comunicação de dados há mais de seis meses e que, por isso, pudessem trazer maiores contribuições para esta etapa. Além disso, relatórios e outras pesquisas de satisfação, conduzidas pela empresa e pela Agência Nacional de Telecomunicações [Anatel] (2003), também serviram como fonte de informações.

A coleta de dados se deu por meio de entrevistas em profundidade com cada um dos entrevistados. O instrumento de coleta de dados utilizado foi um questionário aberto e não-estruturado, com tópicos que servissem de guia ao entrevistador e proporcionassem maior liberdade de respostas. Os resultados foram categorizados e quantificados para medição dos indicadores (variáveis).

\section{Etapa Descritiva}

A etapa descritiva é de natureza quantitativa e teve por objetivo medir o grau de satisfação dos clientes em relação ao serviço prestado, realizando-se uma survey nessa etapa. 
O instrumento de coleta de dados foi composto por questões que tinham o objetivo de levantar informações sobre o perfil do cliente, sobre os atributos de customização, tempo de entrega e preço e demais variáveis que emergiram da etapa qualitativa. Não foram utilizadas palavras negativas, conforme recomenda Hinkin (1995); optou-se por frases diretas, objetivas e claras (Hayes, 1997). A escala utilizada foi a do tipo Likert de 5 pontos, com o valor 1 igual a "Totalmente Insatisfeito" e o valor 5 igual a "Totalmente Satisfeito". A validade do conteúdo foi feita por especialistas no assunto e também pela realização de um pré-teste com dez clientes. No final, o instrumento ficou composto de 28 questões assim distribuídas: quatro questões sobre o perfil do respondente; uma questão sobre a satisfação geral (variável dependente); 21 questões sobre os atributos do serviço (variáveis independentes); duas questões extras sobre o tema em apreciação.

A população foi definida como todos os clientes empresariais da operadora de telecomunicações no Estado do Rio Grande do Sul com gastos mensais em telecomunicações acima de $\mathrm{R} \$ 500,00$ e que tivessem adquirido um serviço customizado de comunicação de dados nos últimos seis meses. O respondente foi definido como o responsável pela compra e administração deste serviço dentro das empresas, podendo ser desde um diretor de informática até um funcionário de nível operacional. A aplicação do instrumento ocorreu através da Internet. Um e-mail contendo o link para a página da Internet que continha o questionário foi enviado para cada um dos respondentes.

O tamanho da população resultante foi de 2.357 clientes empresariais. Três ondas de e-mails foram enviadas aos possíveis respondentes entre 12 de novembro de 2004 e 5 de janeiro de 2005. A amostra resultante foi de 243 clientes empresariais (taxa de respostas de 10\%). De acordo com a equação para dimensionamento da amostra de populações finitas proposta por Fonseca e Martins (1994, p. 176), o nível de confiabilidade foi de $90 \%$ e erro amostral de $5 \%$.

A análise dos dados foi realizada através do software estatístico SPSS versão 11.0. Foi realizada uma análise fatorial exploratória para redução e sumariação dos dados através do método principal axis factoring e do processo varimax. Assim, diversas variáveis independentes comporiam novas variáveis independentes. Em seguida foi realizada uma análise de confiabilidade através do alfa de Cronbach. As estatísticas descritivas também foram alvo de análise. Por fim, foi aplicada uma análise de regressão múltipla para avaliar a importância de cada uma das variáveis independentes para explicar a variação na variável dependente. 


\section{Resultados}

\section{Etapa Exploratória}

A etapa exploratória buscou identificar os principais indicadores e variáveis que formaram a opinião dos clientes sobre os serviços customizados de comunicação de dados.

Os indicadores (variáveis) foram categorizados e reunidos nos seguintes grupos: atendimento pela equipe de vendas; atendimento prestado pela central de atendimento; serviços de manutenção e reparos; serviços de instalação e correto funcionamento na primeira vez; customização dos serviços; preço e cobrança dos serviços.

Estes indicadores serviram de referência para a elaboração do instrumento de coleta de dados.

\section{Etapa Descritiva}

A etapa descritiva teve por objetivo avaliar o grau de satisfação dos clientes em relação aos serviços de comunicação de dados e também para avaliar a importância de cada atributo do ponto de vista dos clientes.

\section{Caracterização da Amostra}

A amostra final compreendeu 243 clientes empresariais da operadora de telecomunicações no Estado do Rio Grande do Sul. As Tabelas 1 e 2 apresentam os resultados sobre o perfil dos respondentes.

\section{Tabela 1: Setor de Atuação}

\begin{tabular}{l|c|c}
\hline \multicolumn{1}{c|}{$\begin{array}{c}\text { Setor de } \\
\text { atuação }\end{array}$} & $\begin{array}{c}\text { Freqüência } \\
\text { absoluta }\end{array}$ & $\begin{array}{c}\text { Frequiência } \\
\text { relativa }\end{array}$ \\
\hline Indústria & 39 & $16,0 \%$ \\
Comércio & 67 & $27,6 \%$ \\
Serviços & 107 & $44,0 \%$ \\
Serviços Públicos & 11 & $4,5 \%$ \\
Outro & 18 & $7,4 \%$ \\
Omitido & 1 & $0,4 \%$ \\
Total & 243 & $100 \%$ \\
\hline
\end{tabular}

Fonte: Coleta de dados. 


\section{Tabela 2: Quantidade de Funcionários}

\begin{tabular}{l|c|c}
\hline $\begin{array}{c}\text { Quantidade de } \\
\text { funcionários }\end{array}$ & $\begin{array}{c}\text { Frequiência } \\
\text { absoluta }\end{array}$ & $\begin{array}{c}\text { Frequiência } \\
\text { relativa }\end{array}$ \\
\hline Até 50 & 135 & $55,5 \%$ \\
Entre 50 e 99 & 23 & $9,5 \%$ \\
Entre 100 e 499 & 47 & $19,3 \%$ \\
Mais de 499 & 35 & $14,4 \%$ \\
Omitido & 3 & $1,2 \%$ \\
Total & 243 & $100,0 \%$ \\
\hline
\end{tabular}

Fonte: Coleta de dados.

A amostra ficou composta por pequenas empresas (menos de 50 funcionários igual a 55,5\%) embora parte considerável $(35,7 \%)$ também seja composta de empresas com mais de 100 funcionários (Tabela 2). A maioria dos respondentes tinha cargos de direção ou gerência, totalizando 78,3\% da amostra. Quanto ao setor de atuação, a amostra obteve uma concentração de empresas de serviços, seguida de empresas de comércio e da indústria (Tabela 1).

\section{Análise Fatorial Exploratória}

$\mathrm{O}$ teste de Kaiser-Meyer-Olkin [KMO] foi realizado para testar a adequação dos dados para a análise fatorial exploratória. O índice KMO ficou em 0,910, acima do limite aceitável de 0,700 (Hair, Anderson, Tatham, \& Black, 1998), possibilitando assim a aplicação deste teste.

A Tabela 3 apresenta os resultados da matriz fatorial rotada através do processo Varimax. As cargas fatoriais inferiores a 0,400 foram omitidas da matriz. As variáveis de 1 a 4 e de 27 a 29 não entraram na análise, pois não eram variáveis independentes. As variáveis 8, 17 e 23 apresentaram cargas fatoriais em mais de um fator, sendo cortadas das análises posteriores. Identificou-se a formação de cinco construtos que foram nomeados e classificados conforme a Figura 2. 
Tabela 3: Matriz Fatorial Rotada

\begin{tabular}{|c|c|c|c|c|c|}
\hline \multirow{2}{*}{ Variáveis } & \multicolumn{5}{|c|}{ Fatores } \\
\hline & 1 & 2 & 3 & 4 & 5 \\
\hline 5. O atendimento pessoal realizado pelo consultor. & & & 0,598 & & \\
\hline 6. O atendimento realizado pelos funcionários do call center. & & & 0,853 & & \\
\hline 7. A rapidez no atendimento do call center. & & & 0,765 & & \\
\hline 8. A precisão das informações prestadas pelo call center. & 0,401 & & 0,674 & & \\
\hline $\begin{array}{l}\text { 9. O conhecimento e competência dos funcionários da } \\
\text { instalação. }\end{array}$ & 0,596 & & & & \\
\hline 10. A rapidez no tempo de instalação do serviço. & & & & & 0,887 \\
\hline 11. O cumprimento dos prazos de instalação. & & & & & 0,713 \\
\hline 12. O correto funcionamento do serviço logo após a instalação. & 0,594 & & & & \\
\hline $\begin{array}{l}\text { 13. O atendimento e presteza dos funcionários do } \\
\text { reparo/manutenção. }\end{array}$ & 0,740 & & & & \\
\hline 14. A rapidez no tempo de reparo/manutenção do serviço. & 0,674 & & & & \\
\hline 15. Os horários disponíveis para reparo/manutenção no serviço. & 0,507 & & & & \\
\hline 16. O cumprimento dos prazos de reparo/manutenção do serviço. & 0,690 & & & & \\
\hline $\begin{array}{l}\text { 17. A estabilidade/ininterrupção (poucas falhas) do serviço da } \\
\text { prestadora. }\end{array}$ & 0,655 & 0,438 & & & \\
\hline 18. O desempenho da velocidade que foi contratada. & & 0,659 & & & \\
\hline 19. A diversidade de opções de velocidade oferecida. & & 0,716 & & & \\
\hline $\begin{array}{l}\text { 20. A disponibilidade/capilaridade do serviço em diversas } \\
\text { localidades. }\end{array}$ & & 0,724 & & & \\
\hline 21. A correta adequação do serviço às suas necessidades. & & 0,699 & & & \\
\hline 22. O cumprimento das especificações técnicas do serviço. & & 0,507 & & & \\
\hline 23. A modernidade e aparência dos equipamentos. & 0,536 & & & 0,406 & \\
\hline 24. O preço cobrado pela mensalidade do serviço. & & & & 0,592 & \\
\hline 25. As condições contratuais apresentadas. & & & & 0,681 & \\
\hline 26. A correta cobrança dos valores na conta. & & & & 0,628 & \\
\hline
\end{tabular}

Fonte: Coleta de dados.

Figura 2: Caracterização dos Construtos

\begin{tabular}{l|c|c|c|c}
\hline \multicolumn{1}{c|}{ Denominação } & Sigla & Variáveis & Tipo da variável & $\begin{array}{c}\text { Alpha de } \\
\text { Cronbach }\end{array}$ \\
\hline Atendimento & At & 5,6 e 7 & Independente & 0,7577 \\
Confiabilidade & $\mathrm{Cf}$ & $9,12,13,14,15$ e 16 & Independente & 0,8806 \\
Tempo de entrega & $\mathrm{Te}$ & 10 e 11 & Independente & 0,9117 \\
Flexibilidade & $\mathrm{Fx}$ & $18,19,20,21$ e 22 & Independente & 0,8406 \\
Preço & $\mathrm{Pç}$ & 24,25 e 26 & Independente & 0,7355 \\
Satisfação geral & $\mathrm{Sg}$ & 27 & Dependente & ------ \\
\hline
\end{tabular}

Cada um destes construtos pode ser descrito da seguinte forma.

- Atendimento - caracteriza-se pela acolhida prestada aos clientes pela equipe comercial e também pela central de atendimento aos clientes. 
- Confiabilidade - é a segurança para o cliente de que os serviços irão funcionar constantemente e, em caso de falhas, haverá suporte rápido e eficiente, não os deixando sem o serviço.

- Tempo de entrega - é caracterizado como o prazo ou demora que a empresa fornecedora levou para instalar o serviço e se este tempo estava dentro do acordado com o cliente.

. Customização - é o grau em que os clientes percebem que os serviços foram feitos especificamente para eles e estão de acordo com as suas necessidades e desejos.

- Preço - é o construto relacionado às questões de valor, condições de contrato e cobrança dos serviços.

A análise de confiabilidade dos construtos por meio do alfa de Cronbach indicou que todos os construtos apresentaram valores superiores a 0,700 , valor mínimo utilizado como referência (Hair et al., 1998), confirmando suas consistências internas (Figura 2).

\section{Estatística Descritiva}

A estatística descritiva proporcionou uma análise dos construtos observados. A Tabela 4 apresenta os resultados de média, mediana, moda, variância, desviopadrão, assimetria e curtose para cada um dos construtos e para a satisfação geral.

O construto customização $(\mathrm{Cm})$ teve a maior média $(3,6996)$. Esse resultado sugere que o fato de os clientes poderem utilizar um serviço que eles mesmos configuraram é o que mais os deixa satisfeitos. A confiabilidade (Cf) é o segundo item com a maior média de satisfação $(3,3291)$. Isso mostra que os itens relativos a reparos e funcionamento do serviço proporcionam maior satisfação do que os outros construtos. O Atendimento (At), Tempo de entrega (Te) e o Preço (Pç) tiveram médias muito próximas umas das outras. Foi realizado um teste t entre os pares destes construtos para verificar se suas médias apresentavam diferenças significativas. Os resultados indicaram que não havia diferenças significativas entre as médias destes construtos.

Entretanto deve-se destacar que o Tempo de entrega (Te) e o Atendimento (At) tiveram os maiores desvios-padrão. Deste modo, houve considerável variação de respostas dos clientes para estes construtos. 
Tabela 4: Estatística Descritiva dos Construtos e da Satisfação Geral

\begin{tabular}{l|r|r|r|r|r|r}
\hline & \multicolumn{1}{c|}{ At } & \multicolumn{1}{c|}{ Cf } & \multicolumn{1}{c|}{ Te } & \multicolumn{1}{c|}{ Cm } & \multicolumn{1}{c}{ Pç } & \multicolumn{1}{c}{$\mathrm{Sg}$} \\
\hline $\mathrm{N} \quad$ Válidos & 243 & 243 & 243 & 243 & 243 & 243 \\
\multicolumn{1}{c}{ Faltantes } & 0 & 0 & 0 & 0 & 0 & 0 \\
Média & 3,1684 & 3,3291 & 3,1803 & 3,6996 & 3,1382 & 3,2353 \\
Mediana & 3,2289 & 3,3333 & 3,1803 & 3,8000 & 3,2350 & 3,0000 \\
Moda & 3,67 & 3,00 & 4,00 & 4,80 & 3,00 & 4,00 \\
Desvio-padrão & 1,17671 & 0,95701 & 1,29944 & 0,85691 & 0,97070 & 1,09247 \\
Variância & 1,38465 & 0,91587 & 1,68855 & 0,73430 & 0,94225 & 1,19349 \\
Simetria & $-0,073$ & $-0,302$ & $-0,273$ & $-0,451$ & $-0,330$ & $-0,425$ \\
Curtose & $-0,845$ & $-0,449$ & $-0,926$ & 0,398 & $-0,264$ & $-0,378$ \\
\hline
\end{tabular}

Fonte: Coleta de dados.

Uma análise da Satisfação geral $[\mathrm{Sg}]$ mostra que os clientes estão satisfeitos com os serviços da empresa. Embora a média não seja muito alta $(3,2353)$, a maioria das citações foi alta (moda $=4,000)$. Isso indica uma variação nas satisfações dos clientes, fato comprovado pelo valor do desvio-padrão $(1,09247)$. Dessa forma, conclui-se que a empresa apresenta um desempenho variado e não-regular que gera, ao mesmo tempo, satisfação e insatisfação em parte considerável de seus clientes.

Por fim, as medidas de forma alcançaram valores próximos de zero para todos os construtos, evidenciando efetiva distribuição normal e quase simétrica dos dados.

\section{Análise de Regressão Múltipla}

Esse procedimento teve como objetivo avaliar a importância relativa de cada atributo do ponto de vista dos clientes. O método utilizado foi o de entrada de todas as variáveis independentes (Hair et al., 1998). Nesse caso os construtos identificados mediante a análise fatorial exploratória. $\mathrm{O}$ modelo de regressão analisado explica $70 \%$ da variação na variável dependente $\left(\mathrm{r}^{2}=0,714\right)$ e os resultados do teste F (Sig. $=0,000$ ) atestaram a significância do modelo (Garson, 2004).

A Tabela 5 apresenta os coeficientes padronizados do modelo de regressão utilizado. Assim, é possível identificar a importância relativa de cada construto para explicar a variação na satisfação geral dos clientes. 


\section{Tabela 5: Coeficientes do Modelo de Regressão}

\begin{tabular}{c|c|c|c|c|c|c|c}
\hline \multirow{2}{*}{ Modelo } & \multicolumn{2}{|c|}{$\begin{array}{c}\text { Coeficientes não } \\
\text { padronizados }\end{array}$} & $\begin{array}{c}\text { Coeficientes } \\
\text { padronizados }\end{array}$ & \multirow{2}{*}{$\mathrm{t}$} & \multirow{2}{*}{ Sig. } & \multicolumn{2}{|c}{$\begin{array}{c}\text { Estatísticas de } \\
\text { colinearidade }\end{array}$} \\
\cline { 2 - 4 } & $\mathrm{B}$ & Erro-padrão & Beta & & & $\begin{array}{c}\text { Nível de } \\
\text { tolerância }\end{array}$ & VIF \\
\hline 1 (Constante) & $-0,438$ & 0,176 & & $-2,497$ & 0,013 & & \\
At & 0,244 & 0,043 & 0,263 & 5,618 & 0,000 & 0,552 & 1,812 \\
$\mathrm{Cf}$ & 0,414 & 0,063 & 0,363 & 6,595 & 0,000 & 0,398 & 2,510 \\
$\mathrm{Te}$ & 0,074 & 0,039 & 0,089 & 1,934 & 0,054 & 0,574 & 1,743 \\
$\mathrm{Cm}$ & 0,165 & 0,059 & 0,129 & 2,801 & 0,006 & 0,565 & 1,769 \\
$\mathrm{Pc}$ & 0,215 & 0,053 & 0,191 & 4,051 & 0,000 & 0,544 & 1,838 \\
\hline
\end{tabular}

Fonte: Coleta de dados.

Os resultados mostram que o construto confiabilidade $(\mathrm{Cf})$ é o que tem a maior importância relativa no modelo proposto, explicando 36,3\% da variação na variável dependente. Em seguida aparece Atendimento (At), Preço (Pç) e Customização (Cm), com importâncias relativas de, respectivamente, 26,3\%, 19,1\% e 12,9\% para explicar as variações na variável Satisfação geral $(\mathrm{Sg})$. O construto Tempo de entrega (Te) foi o que apresentou a menor importância relativa, com apenas 8,9\%.

Uma análise dos valores do nível de tolerância e VIF (Tabela 5) mostra um nível de multicolinearidade entre as variáveis consideradas aceitáveis (Garson, 2004; Hair et al., 1998), sem repercussões negativas para as análises dos dados.

Portanto a confiabilidade é o item com maior importância relativa para a satisfação geral. Em seguida aparecem, em ordem de importância, atendimento, preço, customização e tempo de entrega. Ao comparar esses resultados com as médias que cada construto obteve, observa-se que a confiabilidade teve a segunda maior média e é o que mais contribui para a satisfação geral. A customização, apesar de ter a maior média de satisfação, contribui pouco para a satisfação geral dos clientes $(12 \%)$. O contrário ocorre com os construtos atendimento e preço, que tiveram médias baixas, mas contribuem significativamente para a satisfação geral: $26 \%$ e $19 \%$ respectivamente. Por fim, o tempo de entrega obteve a menor importância relativa para explicar a variação na satisfação geral dos clientes.

É importante considerar também algumas limitações do trabalho. Uma delas refere-se à avaliação dos serviços customizados de apenas uma operadora de telecomunicações. Isso pode causar impacto nos resultados, pois estuda problemas concernentes a apenas uma empresa. Outra limitação diz respeito ao número de construtos analisados e, por conseqüência, de atributos avaliados pelos clientes, ou seja, a pesquisa avaliou cinco atributos (critérios competitivos), embora outros atributos também pudessem ser avaliados. 
Além do mais, esta pesquisa avaliou a importância dos trade-offs a partir da satisfação dos clientes com o desempenho dos atributos dos serviços prestados pela empresa. A avaliação ocorreu após a compra e o uso do serviço, o que pode influenciar o julgamento dos clientes, pois as experiências com o serviço alteramse ao longo do tempo. É o caso, por exemplo, da avaliação em relação ao tempo de entrega. Por ser evento que ocorre em um dos primeiros momentos na relação com o cliente, este pode subestimar tal experiência em face de outras mais recentes.

\section{Análise e Discussão}

\section{A Inexistência do Trade-off entre Customização e Tempo de Entrega?}

A revisão da literatura destacou a incompatibilidade de elevado desempenho simultâneo entre os critérios competitivos customização e tempo de entrega, gerando um possível trade-off operacional. Porém, diferentemente disso, uma análise dos resultados encontrados com o uso da análise de regressão múltipla sugere que o tempo de entrega não apresenta a mesma importância que a customização para explicar a satisfação geral dos clientes.

Além do mais, uma análise das estatísticas descritivas ajuda a compreender melhor esse resultado. A customização teve a maior média de satisfação $(3,6996)$, enquanto o tempo de entrega obteve a terceira maior média $(3,1803)$. Um teste de médias entre estes atributos mostrou que eles são significativamente diferentes $($ Sig. $\mathbf{= 0 , 0 0 0}$ ). Estes resultados mostram que os clientes apresentavam maior satisfação com a customização do que com o tempo de entrega. Concomitantemente, o tempo de entrega obteve mais que o dobro de variância do que a customização $(\mathrm{Te}=1,6885$ e $\mathrm{Cm}=0,7343)$. A variação maior das respostas do atributo tempo de entrega mostra que há maior quantidade de clientes insatisfeitos em relação a este atributo. Entretanto a variação de respostas em torno da satisfação média do tempo de entrega não foi suficiente para afetar a satisfação geral dos clientes, evidenciando a pouca importância deste atributo. Como consequiência, constata-se que o trade-off entre customização e tempo de entrega é pouco relevante no caso analisado.

Uma possível explicação para este resultado pode ser atribuída à natureza de ambos os atributos. O tempo de entrega é atributo que ocorre em momento específico da relação entre a operadora de telecomunicações e o cliente. 
Geralmente, a entrega do serviço ocorre no início dessa relação. Por outro lado, a customização é um item que está diariamente sendo percebido pelo cliente, como, por exemplo, na transmissão de suas informações entre suas diversas unidades. Assim, caso o serviço de transmissão de dados esteja lento ou fora das especificações apresentadas, o cliente poderá verificar tais alterações no serviço contratado. Dessa forma, a experiência com o tempo de entrega do serviço pode perder importância ao longo do tempo, em face de outras experiências que podem vir a ocorrer com o desempenho do serviço.

Além disso, a instalação e os testes de equipamentos necessários para a entrega dos serviços de telecomunicações tendem a ser problemáticos, devido a questões de infra-estrutura de redes e cabos nas ruas. Após a resolução dessas questões e efetiva entrega do serviço, a quantidade de problemas tende a reduzir-se.

Um dos reflexos desse resultado para a área de operações da empresa é o questionamento sobre a necessidade de se incorrer no trade-off entre customização e tempo de entrega. Se os clientes não atribuem a mesma importância para esses atributos, por que a área de operações teria que proporcionar o mesmo desempenho para ambos? Não seria mais prudente proporcionar elevado desempenho àquele atributo que tem maior importância para os clientes?

Tais questionamentos encontram suporte teórico na proposição levantada por Silveira e Slack (2001), em que as expectativas e exigências dos clientes são determinantes para a formação dos trade-offs. Collins et al. (1998) também dividem essa opinião, ao destacarem a importância dos clientes para o desempenho dos critérios competitivos da área de operações.

\section{O Trade-off entre Customização e Preço}

Alinhados com o proposto pela literatura, os resultados encontrados confirmam o trade-off entre customização e custos, pois o preço teve importância relativa maior do que a customização para explicar a satisfação dos clientes, enquanto o preço teve uma importância de $19,1 \%$, a customização obteve $12,9 \%$.

Novamente, uma análise das estatísticas descritivas destes atributos reforça essa constatação. A customização teve a maior média de satisfação $(\mathrm{Cm}=3,6996)$ e o preço uma das menores $(\mathrm{Pç}=3,1382)$. Um teste de médias entre estes atributos mostrou que eles são significativamente diferentes (Sig. $=0,000)$. Os índices de variância dos dois atributos são próximos $(\mathrm{Cm}=0,73430$ e Pç $=0,94225)$, o que mostra que houve pouca variação em torno das respostas encontradas. Assim, embora os clientes apresentem a tendência de estarem mais satisfeitos 
com a customização, a satisfação ou insatisfação com o preço tem maior importância para os clientes.

A questão que envolve preços e custos é sempre importante para as empresas, tendo em vista a necessidade atual que elas têm de reduzir seus custos operacionais. Além disso, os clientes analisam o preço dos serviços sempre que os pagam à operadora de telecomunicações e possivelmente fazem comparações com os serviços prestados pelos concorrentes.

Esses resultados mostram que os clientes podem estar satisfeitos com a customização, mas também atribuem elevada importância ao preço do serviço prestado. Para a organização, significa que há a necessidade da área de operações proporcionar elevado desempenho simultâneo nos atributos customização e custos, indicando assim a necessidade de minimizar o tradeoff existente.

\section{O Papel dos Clientes na Determinação dos Trade-offs}

A análise dos resultados sinaliza também a importância do papel dos clientes na determinação dos trade-offs operacionais. Sabe-se que o surgimento dos tradeoffs ocorre, caso a área de operações estabeleça uma estratégia que priorize o elevado desempenho simultâneo em um par ou conjunto de critérios. Uma análise dos resultados indica, entretanto, que isso tem sentido somente se o mercado, através dos clientes, atribuir uma mesma importância a dois ou mais critérios que usualmente sejam incompatíveis. Por exemplo, se os clientes atribuírem uma elevada importância à alta variedade de produto a preço baixo; então a área de operações poderá incorrer em um trade-off entre estes critérios, pois haverá necessidade de elevado desempenho simultâneo em customização e custos. Por outro lado, se os clientes atribuírem elevada importância à customização e baixa importância ao tempo de entrega, então não há razão em incorrer no trade-off, pois a área de operações não precisaria buscar elevado desempenho simultâneo nestes critérios.

Desse modo, os clientes são determinantes para as ocorrências dos trade-offs dentro da área de operações, assim como destaca Silveira e Slack (2001). Aárea de operações deverá dar maior ênfase aos critérios e atributos que os clientes valorizarem, quer isso implique em trade-offs ou não. Logo, o gerenciamento dos trade-offs também está diretamente relacionado com as expectativas e necessidades dos clientes. 


\section{ConClusões}

Esta pesquisa teve como objetivo avaliar as relações entre os atributos customização, tempo de resposta e preço do ponto de vista dos clientes. Para isso buscou-se identificar a importância atribuída pelos clientes a estes atributos mediante uma pesquisa de satisfação, a partir da avaliação de seus desempenhos. Uma das contribuições teóricas, portanto, emerge dos resultados encontrados. Foi verificado que os clientes não atribuíram a mesma importância aos critérios competitivos customização e tempo de entrega, o que faz com que a área de operações, no caso analisado, não precise incorrer em um trade-offentre estes atributos, contrariamente ao que propõe a literatura. Por outro lado, a importância simultânea atribuída à customização e ao preço indicaria a necessidade da área de operações em incorrer no trade-off entre customização e custos, buscando formas de minimizá-lo.

Outra contribuição teórica deste trabalho está na inclusão do cliente na discussão relativa aos trade-offs, proporcionando novas perspectivas e alternativas para o gerenciamento desse recorrente problema gerencial em operações. Além disso, da mesma forma que o modelo do "cone de areia" de Ferdows e De Meyer (1990) lança dúvidas sobre a existência dos trade-offs, este trabalho questiona a necessidade da ocorrência dos trade-offs entre alguns critérios competitivos, quando o cliente é investigado.

Novos trabalhos poderão avaliar o ponto de vista de clientes de outros produtos e serviços customizados, bem como outros trade-offs existentes na área de operações, com o objetivo de validar tal proposição metodológica. Outras pesquisas também podem avaliar a importância dos critérios competitivos antes da compra do serviço pelo cliente, com o objetivo de avaliar suas preferências e expectativas e não apenas após o uso.

\section{Artigo recebido em 23.03.2006. Aprovado em 19.06.2006.}

\section{ReferênCIAs Bibliográficas}

Agência Nacional de Telecomunicações. (2003).

Pesquisa de satisfação de clientes de serviços telefônicos. Recuperado em 27 junho, 2004, de http:// www.anatel.gov.br/Portal/documentos/ biblioteca/publicacao/pesquisa/
Ahlström, P., \&

Westbrook, R. (1999).

Implications of mass customization for operations management: an exploratory survey. International Journal of Operations \& Production Management, 19(3), 262-275. 
Bardacki, A., \&

Whitelock, J. (2003).

Mass-customization in marketing: the customer perspective. Journal of Consumer Marketing, 20(4-5), 463479.

Boyer, K. K., \&

Lewis, M. W. (2002).

Competitive priorities: investing the need for trade-offs in operations strategy. Production and Operations Management, 11(1), 9-20.

Broekhuizen, T. L. J., \&

Alsem, K. J. (2002).

Success factors for mass customization: a conceptual mode. Journal of MarketFocused Management, 5(4), 309-330.

Collins, R. S.,

Cordon, C., \&

Julien, D. (1998).

An empirical test of the rigid flexibility model. Journal of Operations Management, 16(2-3), 133-146.

Corbett, C., \&

Wassenhove, L. (1993).

Trade-offs? What trade-offs? Competence and competitiveness in manufacturing strategy. California Management Review, 35(4), 107-122.

Ferdows, K., \&

De Meyer, A. (1990).

Lasting improvements in manufacturing: in search of a new theory. Journal of Operations Management, 9(2), 168-184.

Fonseca, J. S., \&

Martins, G. A. (1994).

Curso de estatística (5a ed.). São Paulo: Atlas.
Garson, D. (2004).

PA 765 statnotes: an online textbook. Recuperado em 13 novembro, 2004, de http://www2.chass.ncsu.edu/ garson/pa765/statnote.htm

Hair, J. F., Jr.,

Anderson, R. E.,

Tatham, R. L., \&

Black, W. (1998).

Multivariate data analysis (5a ed.). Upper Saddle River, NJ: Prentice Hall.

Hayes, B. E. (1997).

Measuring customer satisfaction: survey design, use, and statistical analysis methods (2a ed.). Milwaukee: ASQ Quality Press.

Hayes, R. H., \&

Pisano, G. P. (1996).

Manufacturing strategy: at the intersection of two paradigm shifts. Production and Operations Management, 5(1), 25-41.

Heikkilä, J. (2002).

From supply to demand chain management: efficiency and customer satisfaction. Journal of Operations Management, 20(6), 747-767.

Hinkin, T. R. (1995).

A review of scale development practices in the study of organizations. Journal of Management, 21(5), 967988.

McCutcheon, D. M.,

Raturi, A. S., \&

Meredith, J. R. (1994).

The customizations-responsiveness squeeze. Sloan Management Review, 35(2), 89-99. 
Mapes, J.,

New, C., \&

Szwejczewski, M. (1997).

Performance trade-offs in manufacturing plants. International Journal of Operations \& Production Management, 17(10), 1020-1033.

New, C. (1992).

World-class manufacturing versus strategic trade-offs. International Journal of Operations \& Production Management, 12(6), 19-31.

Noble, M. A. (1995).

Manufacturing strategy: testing the cumulative model in a multiple country context. Decision Sciences, 26(5), 693-721.

Paiva, E. L.,

Carvalho, J. M. J., \&

Fensterseifer, J. A. (2004).

Estratégia de produção e de operações. Porto Alegre: Bookman.

Pine, B. J. (1994).

Personalizando produtos e serviços: customização maciça. São Paulo: Makron Books.

Rohr, S. S., \&

Corrêa, H. L. (1998).

Time-based competitiveness in Brazil: whys and hows. International Journal of Operations \& Production Management, 18(3), 233-245.

Salvador, F., \&

Forza, C. (2004).

Configuring products to address the customization-responsiveness squeeze: a survey of management issues and opportunities. International Journal of Production Economics, 91(3), 273-291.

Schmenner, R. W., \&

Swinl, M. L. (1998).

On theory in operations management. Journal of Operations Management, 17(1), 97-113.

Silveira, G., \&

Fogliatto, F. S. (2002).

Modelo acumulativo de prioridades competitivas: validação teórica e impacto em performance. Produto \& Produção, 6(2), 1-11.

Silveira, G., \&

Slack, N. (2001).

Exploring the trade-off concept. International Journal of Operations \& Production Management, 21(7), 949-964.

Skinner, W. (1969).

Manufacturing - the missing link in corporate strategy. Harvard Business Review, 47(3), 5-14.

Skinner, W. (1974).

The focused factory. Harvard Business Review, 52(3), 113-121.

Slack, N.,

Chambers, S.,

Harland, C.,

Harrison, A., \&

Johnston, R. (1999).

Administração da produção. São Paulo: Atlas.

Stalk, G. J. (1998).

Time - the next source of competitive advantage. Harvard Business Review, 66(4), 41-51. 
Szwejczewski, M.,

Mapes, J., \&

New, C. (1997).

Delivery and trade-offs. International Journal of Production Economics, 53(3), 323-330.

Swaminathan, J. M. (2001).

Enabling customization using standardized operations. California Management Review, 43(3), 125-135.

Verma, R., \&

Thompson, G. M. (1999).

Managing service operations based on customer preferences. International Journal of Operations \& Production Management, 19(9), 891-905.
Wheelwright, S. C. (1984).

Manufacturing strategy: defining the missing link. Strategic Management Journal, 5(1), 77-91.

Wheelwright, S. C., \&

Clark, K. B. (1992).

Competing through development capability in a manufacturing-based organization. Business Horizons, 35(4), 29-43.

Wheelwright, S. C., \&

Hayes, R. H. (1985).

Competing through manufacturing. Harvard Business Review, 63(1), 99109. 